\title{
Rotation, Convection, Activity and Lithium in Single G and $\mathrm{K}$ giants
}

\author{
Renada K. Konstantinova-Antova \\ Institute of Astronomy, Bulgarian Academy of Sciences, 72 Tsarigradsko \\ shosse blvd., BG-1784 Sofia, Bulgaria, e-mail: antovi@astro.bas.bg
}

\begin{abstract}
We studied the activity and evolutionary stage of 14 low and intermediate mass single giants that should meet the conditions for dynamo action and Li-enrichment. Only small part of them exhibit either moderate to fast rotation and evidences for magnetic activity and/or high $\mathrm{Li}$ abundance. There are also intermediate-mass giants with fast rotation, but no evidences for magnetic activity was found in them and their $\mathrm{Li}$-content is low one. On the other hand, no fast rotation and activity were observed in some of the Li-rich giants. There is a large difference in the activity properties and $\mathrm{Li}$ content in stars with similar masses and evolutionary stage.
\end{abstract}

\section{Introduction}

The recent theoretical studies point out that similar conditions for transport of freshly synthesized lithium and fast rotating material from the interior should take place in the stars on the red giant branch (RGB). The aim of our work was to examine the predictions about the link among the rotation, $\mathrm{Li}$ content and activity in the RGB stars.

\section{Methods of the Study}

For this purpose $14 \mathrm{G}$ and $\mathrm{K}$ single giants for which observational and theoretical premises for activity exist were selected. Stars with reported spectral or photometric activity indicators (HD9746, HD33798, V654 Her, $\nu$ Oph and $\tau$ CrB) are among them. Stars with faster rotation (HD112989, HD121107, HD145001 and HD112989) and Li-rich ones (HD112127, HD108471, HD148293 and HD183492) were studied, too.

A study of the time behavior of spectral and photometric activity indicators was done for these stars in the period 1993-2001. The spectral observations were carried out in the lines CaII $\mathrm{K} \& \mathrm{H}$ and $\mathrm{H}_{\alpha}$. Available literature data for the $\mathrm{X}$-ray emission of the sample stars were also taken into account. The study of the evolutionary stage was done by means a determination of their location on the $\mathrm{H}-\mathrm{R}$ diagram on the basis of Hipparcos parallaxes and evolutionary tracks of Schaller et al. (1992). The analysis of the literature data for C,N,O and $\mathrm{Li}$ abundances and the ratio ${ }^{12} \mathrm{C} /{ }^{13} \mathrm{C}$ allowed us to determine whether these stars are evolved after the first dredge-up phase. Their $v \sin i$ data are also analysed and compared with the evolutionary stage. For this purpose data published in 
De Medeiros \& Mayor (1999) and tracks of the rotational evolution for low and intermediate mass giants presented in Pasquini et al. (2000) were used.

For more information about the sample stars and the applied methods we refer to Konstantinova-Antova (2001).

\section{Results and Conclusions}

The results of our study are summarized as follows:

- Among 14 giants we studied only 4 (HD9746, HD33798, HD112989 and HD145001) exhibited clear evidences for activity and another one (HD121107) is suspected in it. The first 2 of them are low-mass fairly evolved stars. The rest ones are of intermediate mass, at different stages of their evolution - near and after the base of the RGB. The low-mas active giants deviate significantly from the rotational law for their evolutionary stage, while among the intermediatemass ones only HD112989 deviates slightly. A surprising result was the full lack of activity signatures in the intermediate-mass fast rotating $(v \sin i=9.9$ $\mathrm{km} \mathrm{s}^{-1}$ ) giant HD185958 which appeared evolved after first dredge-up phase, too. The low-mass active giants, in general have enchanced $\mathrm{Li}$-content (Fekel \& Balachandran 1993), while the intermediate-mass ones we studied possess a low one, in agreement with the predictions of Charbonnel \& Ballachandran (2000).

- Most of the studied Li-rich single giants exhibited slow rotation and no evidences for activity. These are stars of similar mass interval $(1-2.5 \mathrm{M} \odot)$ and evolutionary stage like the active ones $\left(1.5-2 \mathrm{M}_{\odot}\right)$.

The faster rotation of the low-mass active giants could be due to angular momentum transport from the interior, while the rotational behavior of the intermediate-mass ones we studied could be explained in the terms of the evolution of rotation with the age, presented in Pasquini et al. (2000).

The small number single active giants and the fact that most of them are fairly evolved ones led us to the conclusion that specific factors should play role for an efficient dynamo action in them. The fact that most low-mass active giants are also Li-rich could be explained by involving angular momentum and chemical elements transport mechanisms, taking into account the evolution of the convection zone on the RGB. The similarity of the evolutionary stage and mass interval between the low-mass active giants and the $\mathrm{Li}$-rich inactive ones could not be easily explained by the theory yet. A deeper insight on the processes in the giant's interiors, and especially those in the boundary layer below the convection zone, is needfull.

\section{References}

Charbonnel C., Balachandran S.C. 2000, A\&A 359, 563

De Medeiros J. R., Mayor M. 1999, A\&AS 139, 433

Fekel F. C., Balachandran S. 1993, ApJ 403, 708

Konstantinova-Antova R. K. 2001, Ph.D. Thesis

Pasquini L., De Medeiros J.R., Girardi L. 2000, A\&A 361, 1011

Schaller G., Schaerer D., Meynet G., Maeder A. 1992, A\&AS 96, 269 